\title{
Scuola e famiglie immigrate: Uno studio in una scuola del Sud Italia ${ }^{1}$
}

\section{Escuelas y familias inmigrantes: un estudio en una escuela en el Sur de Italia}

\section{Schools and Migrant Families: A Case Study in a School in the South of Italy}

\author{
Marco Centorrino*2 \\ centorrinom@unime.it, https://orcid.org/0000-0003-0546-5715 \\ *Università degli Studi di Messina, Italia \\ LiLIA Pellegrino** \\ pellegrino.lilia@alice.it, https://orcid.org/0000-0003-2145-192X \\ ** Universidad de Granada, España
}

\begin{abstract}
Resumen:
Con esta contribución se pretende investigar, en la dimensión de la vida cotidiana, uno de los aspectos a través del cual, en nuestra opinión, es posible analizar la dinámica de integración de los inmigrantes en Italia.

De hecho, la creciente presencia en las últimas décadas de hijos de familias inmigrantes en la escuela italiana ha puesto de manifiesto la necesidad de una comunicación e interacción constantes y efectivas entre la familia y la escuela. La contribución se centra en los primeros resultados de un estudio, dedicado precisamente a este aspecto, Ilevado a cabo en una ciudad del Sur de Italia, en una escuela secundaria que cada año registra un aumento significativo de alumnos de origen extranjero.
\end{abstract}

\begin{abstract}
:
Our contribution intends to investigate, in the dimension of everyday life, one of the aspects through which - in our opinion - it is possible to analyze the dynamics of integration of immigrants in Italy.

In fact, the increasing presence in recent decades of children of immigrant families within the Italian school has highlighted the need for constant and effective communication and interaction between the family and the school. The contribution focuses on the first results of a study, dedicated precisely to this aspect, carried out in a city in Southern Italy, in a lower secondary school that every year records a significant increase in pupils of foreign origin.

Our work analyzes the perceptions of the teaching staff regarding the involvement of
\end{abstract}

1 Il lavoro è frutto di una riflessione comune tra gli autori. Tuttavia, sono da attribuire in via esclusiva a Marco Centorrino I'Introduzione, il paragrafo Metodologia e le Conclusioni; a Lilia Pellegrino i par. Lo stato dell'arte e Risultati.

2 Dirección para correspondencia (correspondence address):

Marco Centorrino. Università degli Studi di Messina. Piazza Pugliatti, 1. 98122 Messina (Italia). 
Nuestro trabajo analiza las percepciones del personal docente con respecto a la participación de las familias inmigrantes en el contexto escolar.

\section{Palabras clave:}

Escuela; familias; inmigración; integración cultural. immigrant families in the school context.

\section{Key words:}

School; families; immigration; cultural integration

\section{Résumé:}

Notre contribution vise à étudier, dans la dimension de la vie quotidienne, l'un des aspects à travers lesquels, à notre avis, il est possible d'analyser la dynamique de l'intégration des immigrants en Italie.

En fait, au cours des dernières décennies, la présence croissante d'enfants de familles immigrées dans l'école italienne a révélé la nécessité d'une communication et d'une interaction constantes et efficaces entre les familles et l'école. La présente contribution porte sur les premiers résultants d'une étude consacrée précisément à cet aspect. La recherche a été menée dans une ville du sud de I'Italie, dans un collège qui enregistre chaque année une augmentation significative des élèves d'origine étrangère.

Notre travail analyse les perceptions du personnel enseignant par rapport à la participation des familles immigrées dans le contexte scolaire.

\section{Mots clés:}

École; familles; immigration; intégration culturelle

Fecha de recepción: 18-11-2019

Fecha de aceptación: 12-1-2020

\section{Introduzione}

Negli ultimi anni la società italiana assiste ad un complesso processo di trasformazione in cui l'immigrazione costituisce un fattore centrale ${ }^{3}$ che ha coinvolto numerose sfere della vita sociale, richiamando l'attenzione di tutte le istituzioni. La scuola è sicuramente una di quelle più interessate nella ricerca di nuove strategie per gestire i cambiamenti generati dalla multiculturalità, anche per via della presenza sempre più cospicua di allievi figli di immigrati ${ }^{4}$. Inoltre, l'ingresso degli alunni di famiglie di origine immigrata ha fatto ulteriormente emergere l'importanza di stabilire punti di interazione e comunicazione tra la famiglia e la scuola. "La

3 Tra il 2001 e il 2011 si è passati da un milione e mezzo di immigrati residenti ai 4,5 milioni, Secondo le stime più recenti, si è adesso giunti a poco meno di 6 milioni (De Santis e Strozza, 2017). Nel 2016 i bambini figli di stranieri nati in Italia sono stati quasi 70 mila, cioè il $14,7 \%$ di tutte le nascite registrate nel Paese, ma se si considerano i nati da madri straniere, la percentuale sale al 19,7 (Bonifazi, 2017).

4 In Italia, si è passati dai 196.414 alunni non italiani dell'anno scolastico 2001-2002 (il 2,2\% della popolazione scolastica) agli802.844 del 2015-2016 (9\%) (Dandolo, 2018). 
dimensione relazionale costituisce il motore di un processo di rinnovamento, I'elemento fondante di ogni progetto educativo interculturale volto a far sviluppare in tutti gli allievi e nelle loro famiglie immigrate il sentimento di una appartenenza plurima che permette di vivere in maniera armoniosa nelle società multietniche come sono quelle attuali" (Silva, 2009, p. 24).

Tuttavia, la scuola non può essere considerata l'unica istituzione delegata all'educazione di bambini e ragazzi; allo stesso tempo, non si può pensare che la famiglia sia la sola responsabile di questo processo. Entrambe sono realtà essenziali nei processi di socializzazione ${ }^{5}$ di bambini e ragazzi ${ }^{6}$. Questa situazione è ancora più importante e spesso difficile da gestire nei riguardi di un bambino/ragazzo straniero immigrato che, accompagnato dalla sua famiglia, attraverso la scuola sperimenta il suo primo contatto con il Paese di accoglienza. In questa fase, la scuola ha un ruolo fondamentale nel sostenere i processi di integrazione nella nuova realtà, sia nei confronti delle famiglie, sia nei riguardi degli studenti e potrebbe essere molto efficace se riuscisse a fare da "ponte"tra la cultura del Paese di origine e quella del Paese di accoglienza. Pertanto, il coinvolgimento delle famiglie immigrate in ambito scolastico potrebbe portare dei benefici per i figli che si ritrovano immersi in una cultura per loro straniera (Bolognesi, 2016). Secondo Cartei (2008), i genitori sono la risorsa fondamentale per il raggiungimento della riuscita scolastica: le diverse culture di appartenenza impongono alla scuola, quindi, di individuare gli strumenti migliori di dialogo e la condivisione delle scelte educative.

La nostra ricerca intende indagare lo stato delle relazioni famiglia-scuola, soffermandosi soprattutto sull'analisi della percezione da parte del corpo docente riguardo la partecipazione delle famiglie immigrate. Come punto di osservazione è stata scelta una scuola secondaria di primo grado di una città del Sud Italia, Messina ${ }^{7}$, caratterizzata da una

5 Da intendere, ovviamente, come canale primario di trasmissione della cultura tra le generazioni.

6 Pur se la visione delle responsabilità educative non sempre è condivisa tra la figura dell'insegnante e quella del genitore. Per un approfondimento, si veda Molinari L. e Bertocchi S. (2007),

7 Messina è sicuramente protagonista della crescente multi-etnicità che ormai caratterizza il Paese. La crescita della popolazione straniera residente sul territorio messinese è notevolmente cambiata in un lasso temporale di dieci anni.

Se nel $1^{\circ}$ gennaio del 2008 gli stranieri residenti sul territorio erano in totale 6.991 (il $2,9 \%$ della popolazione residente), il $1^{\circ}$ gennaio del 2018 i numeri sono quasi 
cospicua presenza di alunni stranieri di origine immigrata. Ovviamente, quello che emerge non può essere definito un quadro omnicomprensivo, né l'oggetto della ricerca assume la valenza di un campione rappresentativo. Tuttavia, il nostro può essere interpretato come uno studio pilota, in grado di contribuire a tracciare delle linee guida per un'indagine su larga scala, che possa tra l'altro prevedere un'adeguata destrutturazione dell'analisi rispetto ai Paesi di provenienza degli immigrati.

\section{Stato dell'arte}

Nella correlazione famiglia-scuola il corpo docente riveste ovviamente un ruolo molto importante. Non deve fare solo in modo di sviluppare, sollecitare e stimolare la partecipazione delle famiglie dei propri alunni, ma anche di stabilire una buona comunicazione con queste ed ottenere risultati positivi e utili per lo sviluppo della formazione degli studenti.

Tuttavia, nel rapporto con i genitori immigrati gli insegnanti si misurano spesso con stili educativi diversi dai propri, con modelli e comportamenti dei ragazzi che non riescono a decodificare, con difficoltà comunicative inedite. Sicuramente è opportuno affermare che la vita che queste famiglie affrontano è piuttosto complessa e in molti casi confluiscono una serie di fattori che non facilitano il loro lavoro educativo e, conseguentemente, incidono nella relazione tra le famiglie e i docenti.

Tutto ciò all'interno di un quadro in cui I'inserimento di questi studenti è spesso problematico: "i dati del MIUR manifestano casi di ripetenza assai più elevati rispetto agli alunni italiani, con una tendenza di gran lunga più palese nel procedere dalle elementari alle secondarie di

raddoppiati: gli stranieri che vivono in città sono 11.885 (il 5,1\% della popolazione residente).

Per quanto riguarda le loro provenienze, l'Asia (58,4\%) è il continente dal quale di origine della maggior parte di essi, seguono I'Europa (25,04\%) e I'Africa (14,2\%). $C^{\prime}$ è anche una minoranza di persone provenienti dal continente americano $(1,9 \%) \mathrm{e}$ dall'Oceania $(0,03 \%)$.

Adesso, come dieci anni fa, la comunità straniera più numerosa è quella formata da individui originari dello Srilanka con il 32,9\% di tutti gli stranieri presenti sul territorio messinese. Al secondo posto la comunità dei filippini: nel 2008 rappresentavano il $26,2 \%$ della popolazione straniera e oggi sono scesi al 19,8\%, seguono la Romania $(12,9)$, il Marocco $(9,7)$, la Grecia $(3,3 \%)$, la Repubblica popolare Cinese $(3,2 \%)$, la Polonia $(2,6 \%)$ e la restante percentuale $(15,6 \%)$ riguarda altre comunità (Statistiche demografiche, 2018). Dati Istat 2018 (http:// www.tuttiitalia.it/sicilia/38-messina/statistiche). 
secondo grado" (Dandolo, 2018, p. 180). A ciò si aggiungano tassi di dispersione scolastica più alti rispetto agli alunni italiani e problematiche peculiari riguardo a determinate discipline (Di Bartolomeo, Bonifazi e Strozza, 2017). Di contro, però, la "maggioranza dei genitori immigrati [...] considera la possibilità di mandare i figli a scuola come uno degli aspetti più positivi della propria vita in Italia: la scuola come veicolo di promozione sociale" (Abis, 2004, p. 426).

I fattori che condizionano la comunicazione diretta tra gli insegnanti e le famiglie sono in particolare quelli interpersonali. Questa "comunicazione include le capacità di ascolto, di scambio e condivisione delle informazioni, così come la capacità di collaborazione attorno a dei compiti specifici" (Granata, Mejri e Rizzi, 2015, p. 79). Tuttavia le famiglie non sempre comprendono le richieste della scuola, non hanno spesso chiari i suoi obiettivi e le sue finalità educative e tendono a vederla come luogo di mera trasmissione di conoscenze disciplinari. Per questo, in molti casi, le famiglie delegano alla scuola tutta l'educazione scolastica dei figli (Silva, 2009)."I fattori interpersonali includono anche le emozioni, gli atteggiamenti e gli stili relazionali del genitore o dell'insegnante che possono incidere nella relazione famiglia-professori" (Granata, Mejrie Rizzi, 2015, p.79). L'atteggiamento distaccato da parte della maggior parte delle famiglie immigrate si deve soprattutto alla scarsa conoscenza della lingua italiana ${ }^{8}$. Tuttavia, non si tratta solo della difficoltà linguistica, ma in termini più ampi del linguaggio della scuola che ha i suoi codici e un suo vocabolario non sempre facilmente comprensibile (Favaro, 2010). Secondo Grasso (2015, p. 211)"gli impliciti culturali di cui sono densi i modelli educativi attuati e l'indecifrabilità e la specificità estrema del linguaggio utilizzato, talvolta rendono incomprensibile alle famiglie il senso delle azioni e delle scelte formative assunte dalla scuola. Molto spesso l'ambiguità e la difficoltà si annida proprio nel non detto e nel non compreso, rispetto a ciò che su entrambi i fronti sembra ovvio e condiviso". Inoltre, come già

8 Fattore che, ovviamente, incide fortemente anche sul rendimento scolastico dei figli. A tal proposito, va considerato come la procedura di iscrizione nel sistema scolastico italiano di studenti stranieri non sia strettamente vincolata a un determinato grado di conoscenza della lingua, pur essendo prevista una prova di accertamento. Ragion per cui, anche studenti che non parlano l'italiano, vengono inseriti nel sistema e, al più, il loro percorso inizia in una (e non più di una) classe precedente rispetto a quella che sarebbe stata assegnata in base alla corrispondenza del titolo conseguito nel Paese di provenienza. Le scuole, in questo caso, devono prevedere appositi percorsi di recupero. 
sottolineato da altri studi, di fronte a parole quali"portfolio", "scuola primaria", "crediti formativi", o addirittura ad acronimi come "pof" o "invalsi", le famiglie sono in difficoltà. Sono termini che sovente comunicano l'idea di un sistema difficile da interpretare, ancor di più per quelle famiglie provenienti da altri Paesi (Zoletto, 2007). "Questa barriera linguistica ha implicazioni emotive sulla relazione tra genitori e insegnanti, in un contesto che favorisce poco o nulla il plurilinguismo e lo scambio comunicativo su più registri linguistici" (Granata, Mejri e Rizzi, 2015, p. 82). Pertanto, esiste un problema di fondo di comunicazione che spesso non viene preso in carico dalla scuola. La barriera linguistica crea, così, incomprensioni "talvolta anche banali, ma che, se protratte nel tempo, degenerano fino a indurre da una parte i genitori ad assumere un atteggiamento distaccato nei confronti delle istituzioni educative e, dall'altra, gli insegnanti a costruirsi una immagine stereotipata delle famiglie immigrate" (Silva, 2009, p. 25).

Ci sono poi fattori strutturali che incidono notevolmente sulla partecipazione di queste famiglie immigrate alla vita della scuola; problematiche che riguardano la loro situazione socio-economica, casi di disagio sociale, nuclei familiari nei quali i genitori non lavorano, condizioni abitative precarie. "Le famiglie immigrate appartengono più facilmente a una classe sociale bassa e questa loro condizione si riversa talvolta anche sulla realtà della scuola e sul rendimento scolastico dei figli" (Granata, Mejrie Rizzi, 2015, p. 83). Sempre tra i fattori strutturali rientrano gli orari di lavoro delle famiglie immigrate(spesso incompatibili con gli orari di apertura della scuola), il loro livello di scolarizzazione insufficiente per poter aiutare il proprio figlio/a nei compiti, nonché l'apatia, il poco interesse verso il percorso scolastico del proprio figlio/a e/o la scarsa motivazione nel partecipare alla vita scolastica non trattandosi di una priorità (Bochaca, 2008). Completano il quadro quei fattori strutturali che si riferiscono"all'assenza di una rete familiare allargata sul territorio, in particolare in un sistema di welfare come quello italiano basato sul sostegno informale della famiglia allargata nella cura dei bambini" (Granata, Mejri e Rizzi, 2015, p. 82).

Infine, i fattori culturali ${ }^{9}$ sono quelli che raggruppano i valori, le visioni e gli immaginari, gli stili di vita e le culture educative, legati in qualche

9 In questo contributo consideriamo come fattori culturali quegli atteggiamenti e quei comportamenti che riguardano le famiglie straniere, secondo l'ottica per cui la cultura non è "solo quella degli altri" (Rogoff, 2003). 
modo alla provenienza culturale sia individuale sia di gruppo che caratterizzano entrambi gli attori famiglia e scuola e che possono influenzare la relazione tra di essi (Granata, Mejri e Rizzi, 2015, p. 79).

Sullo sfondo, va ovviamente considerato il clima in cui avviene l'inserimento delle famiglie straniere nel contesto italiano. Quello dell'immigrazione è ormai - dal punto di vista dei processi culturali e comunicativi - anzitutto un campo di relazioni sempre più saturo e"ad alto voltaggio": una rete di interazioni in fortissimo divenire, la cui intensità energetica è destinata nel tempo ad aumentare quantitativamente e qualitativamente per i singoli e per la collettività. Siamo di fronte a una issue di primaria rilevanza sociale, attorno a cui - non a caso - lo spazio dell'opinione pubblica appare infiammato da orientamenti, punti di vista e polarità divergenti, e rispetto alla quale gli stessi decisori politici continuano a dar prova di clamorose incertezze e contraddizioni nel governare l'ormai irreversibile trasfigurazione multiculturale della società italiana (Carzo, 2011).

\section{Metodologia}

Questo studio si inserisce in una ricerca più ampia, il cui obiettivo è di analizzare e conoscere la partecipazione delle famiglie immigrate in una scuola secondaria della città di Messina, "Manzoni-Dina e Clarenza"10, e capire se questa partecipazione possa dar luogo a processi di integrazione o di esclusione. Il presente contributo analizza la percezione degli insegnanti sulla partecipazione delle famiglie immigrate in ambito scolastico.

10 L'istituto, costituito dalla sede centrale della Scuola Secondaria Manzoni e comprendente il plesso della Scuola Secondaria di Primo Grado "L. Pirandello", accoglie 49 docenti, distribuiti in 19 classi e 7 sezioni. È ubicato in una zona centrale della città. La popolazione scolastica è eterogenea (Tabella 1, in Appendice), solo una piccola parte degli alunni che frequentano l'istituto non risiede nel comprensorio circostante. Il contesto di provenienza risulta vario e, a grandi linee, può essere definito medio. Risultano coesistenti più realtà socio-culturali: accanto alle famiglie pluri-reddito e monoreddito, esiste una realtà di famiglie con inoccupazione giovanile, gruppi di disoccupati, sottoccupati e precari, non mancano casi di povertà.

La carenza di servizi sociali e culturali nella zona, come pure di rilevanti attività produttive, è fonte di un diffuso scontento. Non mancano le risorse, non sempre evidenti e talvolta sopite, in termini di competenze, di tradizioni, di beni storico-culturali, di valori morali e civili, che, nel sinergico impegno delle istituzioni e delle comunità, potranno promuovere concreti processi di sviluppo territoriale, di elevazione culturale e di matura partecipazione civico-sociale. 
Le tecniche utilizzate per raccogliere l'informazione sono state: I'osservazione partecipante realizzata attraverso una tabella di osservazione, la revisione dei documenti e le interviste semi-strutturate, in cui è stato garantito l'anonimato e le cui risposte sono state registrate e trascritte testualmente. In ogni intervista è stato utilizzato un codice identificativo per ciascun docente. Tali interviste si sono svolte una parte all'interno della scuola ${ }^{11}$ e un'altra buona parte all'esterno ${ }^{12}$. Il campione selezionato comprende 36 docenti ( 8 uomini e 28 donne). Si tratta di docenti abilitati, che insegnano nella scuola secondaria di primo grado con anni di esperienza eterogenei (dagli 8 ai 40 anni). II corpo docente intervistato insegna in diverse classi e sezioni ${ }^{13}$ e i loro alunni hanno un'età che oscilla dagli 11 ai 14 anni. La durata di ogni intervista è stata ricompresa tra i 40 e i 60 minuti. Questo tipo di intervista ha permesso ai docenti intervistati di esprimere le loro opinioni, i loro atteggiamenti, le loro preoccupazioni liberamente basandosi sul proprio vissuto, sulle proprie esperienze e dando anche informazioni aggiuntive che sono risultate interessanti ${ }^{14}$.

\section{Risultati}

\section{- La partecipazione familiare a scuola e la barriera linguistica-relazionale}

Dalle interviste ai docenti, emerge innanzitutto la necessità di interesse, dialogo, ascolto, condivisione e coinvolgimento che riguarda entrambe le parti, famiglia e scuola, affinché si generi una relazione positiva che possa indurre ad una partecipazione efficace da parte delle famiglie.

11 Esattamente nell'Auditorium.

12 In un locale pubblico, nei pressi dell'istituto scolastico.

13 Classi I, II e III. Sezioni A/B/C/D/E/F/G.

14 Quattro docenti hanno rifiutato I'intervista, motivando il loro diniego nel seguente modo:

P08: Non ho tempo da dedicare alla tua intervista perché ho altro da fare dopo la scuola! (insegnante di Lettere)

P018: No, assolutamente non se parla, mi vergogno. (insegnante di Tecnologia)

P020: Mi dispiace ma non ho assolutamente tempo da dedicare alla tua ricerca. (insegnante di sostegno)

P026: Scusami, ma ho avuto una serie di impegni e problemi che non mi hanno consentito di presentarmi all'appuntamento. (insegnante di Spagnolo) 
P06: Secondo me queste due importanti istituzioni hanno un obiettivo comune ovvero lo sviluppo e la crescita dell'alunno. È per questo che entrambe devono assolutamente lavorare verso la stessa direzione. Perciò credo che tale unione sia importante, collaborativa e che rappresenti una delle variabili che maggiormente influisce nel rendimento scolastico, soprattutto per gli alunni in fase adolescenziale. (insegnante di Musica)

P07: A parer mio queste due realtà simboleggiano un'alleanza educativa. L'insegnante può conoscere meglio gli alunni soprattutto se ha la possibilità di confrontarsi con i loro genitori. Quindi, tra insegnanti e genitori deve potersi sviluppare un vero patto che consenta ad entrambi di conoscere i percorsi a scuola e a casa dei ragazzi, tanto da poter "costruire" insieme il loro futuro. (insegnante di Francese)

La vita della scuola si sviluppa non solo negli orari di lezione, ma si compone anche di momenti di confronto periodico tra docenti e genitori, oltre a determinati appuntamenti condivisi(come, per esempio, I'elezione dei rappresentanti nei Consigli di Classe e nel Consiglio di Istituto). A tal proposito, una docente (P025) che ha parecchi alunni immigrati in classe, ha raccontato con una punta di amarezza dell'esperienza delle ultime elezioni, in cui per una classe si è registrata la partecipazione di due soli genitori, fatto mai accaduto in passato. Se è vero che un certo numero di famiglie italiane non hanno partecipato, è anche vero che l'assenza delle famiglie straniere è stata dettata dal fatto di essere eccessivamente impegnate in ambito lavorativo. Le parole dell'insegnante fotografano con lucidità la situazione:

P025: All'interno del nostro Consiglio di Istituto, pensavamo di proporre ad alcuni genitori stranieri di entrare a farne parte, perché sono pochissimi quelli che ad oggi ne fanno parte (e sono ancora meno quelli disposti a farne parte). Cercavamo un modo per coinvolgerli maggiormente. Sicuramente dobbiamo ancora capire come vivono e vedono la scuola questi genitori, cosa ne pensano. Coinvolgendoli in un momento di scelta e di decisione, toccherebbero con mano i problemi della scuola. Ma pensare alla loro partecipazione vuole anche dire fornirgli gli strumenti per poter partecipare in modo attivo. Quindi bisogna che conoscano e capiscano la lingua italiana per lo meno discretamente o vanno affiancati da persone che spieghino loro le dinamiche. La faccenda è piuttosto difficile, ma la scuola deve fare qualcosa per avvicinarli e renderli parte integrante di essa. (insegnante d'Italiano) 
Scuola e famiglie immigrate: Uno studio in una scuola del Sud Italia Marco Centorrino

Secondo questa docente e tanti altri, la scarsa presenza dei genitori immigrati è dovuta, in generale, soprattutto alla bassa conoscenza della lingua italiana. Pertanto tutti i professori fanno riferimento alla necessità di fornire maggiori risorse da parte della scuola. Si riferiscono in particolar modo alla necessità di formare persone che svolgano il ruolo di mediatori, per facilitare la comunicazione e la condivisione con e tra le nuove famiglie, dal momento che la maggior parte dei docenti non si sente formata dal punto di vista linguistico e, quindi,avverte limitazioni in ambito comunicativo.

P021: Molte famiglie immigrate non parlano bene l'italiano, bisogna tener conto della lingua o delle lingue di origine, dell'età al momento dell'arrivo in Italia, delle situazioni socio-economiche e, naturalmente, delle motivazioni rispetto alla nuova lingua da apprendere. C'è quindi un problema di comunicazione che non è stato preso in carico dalla scuola. Un corso di italiano per certe famiglie immigrate faciliterebbe sicuramente la loro integrazione e conseguentemente la partecipazione [...]. Tuttavia, la voglia di imparare è rilevante. Purtroppo in questa scuola o, meglio, in tutte le scuole di questa città, è assolutamente assente la figura del mediatore linguistico e culturale. (insegnante di Lettere)

P027, P030: [...] II mediatore sarebbe la figura perfetta che consentirebbe un coinvolgimento maggiore delle famiglie immigrate. (entrambi insegnanti di Religione)

Le molteplici testimonianze in tal senso $^{15}$, rimandano innanzitutto alla visione della comunicazione come sistema relazionale, incentrata sulle

15 Indicative, ad esempio, anche le risposte di altri docenti:

P014: $\grave{E}$ necessario che ci sia un professore o una associazione che favorisca la comunicazione tra la scuola e le famiglie immigrate e che, quindi, abbia una conoscenza sull'interculturalità. (insegnante di Scienze motorie)

P019: Ciò che servirebbe ad agevolare l'integrazione di queste famiglie è la figura del mediatore che manca all'interno della scuola messinese, anche perché noi docenti non abbiamo una preparazione linguistica tale da poterli [riferito ai genitori] accogliere e accompagnare durante il percorso scolastico del proprio figlio. (insegnante di Lettere)

P05: [...] il corpo docente a livello culturale mi sembra abbastanza preparato, ma a livello linguistico potrebbe incontrare serie difficoltà nell'esprimersi e nel capire. lo, per esempio, I'anno scorso ho avuto 2 alunni cinesi, fratello e sorella erano qui da pochissimo. Con i ragazzi usavo un vocabolario cinese, trascrivevo la parola in cinese, scrivevo in italiano e mettevo il simbolo. In Scienze allo stesso modo l'immagine 
tesi della Scuola di Palo Alto. La barriera linguistica incide sui contenuti e questi, a loro volta, sulla relazione. Ciò avviene nonostante molte famiglie, a quanto sembra, mostrino un atteggiamento positivo verso la lingua e la cultura italiana:

P04: Si sforzano sin dal loro primo ingresso a scuola a utilizzare la lingua italiana magari sbagliando, inizialmente monosillabando, ma l'impegno è davvero notevole, anzi spesso si vergognano, nel senso che non si esprimono per paura di sbagliare linguisticamente [...]. (insegnante d'Inglese)

La figura del mediatore potrebbe avere un ruolo sicuramente rilevante, tuttavia - rifacendoci ai risultati raggiunti nel campo degli studi sulla comunicazione interculturale (Gudykunst e Mody, 2002; Baraldi, 2003), occorre intervenire su molteplici fronti. Da un lato, infatti, occorre tenere presente il concetto di competenza comunicativa, cioè il possesso della "sensibilità, le conoscenze e le capacità necessarie per poter interagire efficacemente e in modo appropriato con persone di culture differenti" (Chen e Sarosta, cit. in Mucchi Faina 2006, 102). In questa prospettiva, in termini di effetti, divengono centrali l'incertezza nell'interazione e la miscommunication (gli equivoci che possono influire sull'ordine dell'interazione stessa).

Dall'altro, però, occorre ricordare come - richiamando il modello dinamico di sensibilità interculturale (Bennett, 2002) - l'acquisizione di competenze interculturali richieda tempo e sia strettamente connessa all'esposizione dell'individuo ad esperienze di alterità culturale, ma anche alla sua disponibilità a sviluppare sistemi di valutazione e di osservazione in grado di mutare a seconda della diversità dei contesti. Ciò vale, ovviamente, tanto per gli insegnanti quanto per le famiglie.

È indispensabile completare un percorso che, partendo dalle fasi etnocentriche dell'esperienza, conduca a quelle etnorelative (attraverso, appunto, un modello dinamico). Lo schema che consente di avanzare attraverso le varie fasi prevede il riconoscimento della differenza (accettazione), lo sviluppo di empatia e vicinanza (adattamento) e, alla fine, l'interiorizzazione (integrazione).

e, quindi, la parola in italiano con l'immagine. Poi, un'altra collega ha fatto proprio alfabetizzazione, come se fossero in prima elementare; proprio con le sillabe e le cose, piano piano siamo riusciti a farli esprimere in italiano, diciamo appunto che il primo anno con loro non abbiamo seguito la stessa programmazione. (insegnante di Matematica e Scienze) 


\section{- Il grado di coinvolgimento familiare}

In riferimento al coinvolgimento nella scuola se ne distinguono diversi gradi e forme, come si evince dalle parole dei docenti:

P09: Sicuramente il coinvolgimento è medio-basso per le famiglie immigrate. Pur volendo, non possono collaborare per questioni di tempo, lavoro, e denaro; invece le famiglie italiane sono maggiormente presenti e si lasciano coinvolgere volentieri, ma forse perché hanno più tempo a disposizione $o$ sono aiutate dai parenti. (insegnante di Lettere)

P013: [...] II coinvolgimento complessivamente è medio-alto per le famiglie italiane, ma medio-basso per le famiglie straniere, probabilmente perché possono dedicare meno tempo alla scuola per motivi lavorativi. (insegnante d'Inglese)

P014: Secondo il mio punto di vista, le famiglie immigrate che giungono da noi sono tutte indaffarate, hanno molto lavoro e poco tempo da trascorrere con i propri figli. Ma ci sono quelle famiglie appartenenti ad etnie che tengono maggiormente al percorso scolastico dei propri figli, per cui ci troviamo dinnanzi ad una variegata gamma di famiglie e ad un coinvolgimento eterogeneo. (insegnante di Scienze motorie)

A margine, interessante notare come la variabile degli impegni lavorativi sembri determinare una partecipazione quasi esclusiva delle madri alle riunioni e agli incontri scuola-famiglia ${ }^{16}$, restituendoci un quadro comunque in linea con il quello complessivo del nostro Paese ${ }^{17}$.

$16 \mathrm{Su}$ questo punto, un docente offre una visione alternativa:

P06: Sicuramente la mamma partecipa maggiormente a scuola poiché soprattutto in alcune culture elo per tradizione il marito attribuisce questo ruolo esclusivo alla donna, la quale si interessa di più all'istruzione del proprio figlio o figli probabilmente perché è quella che nutre maggiori aspettative culturali nei riguardi del proprio figlio/i. (insegnante di Musica)

17 Mencarini (2018) rileva che in Italia il "tasso di occupazione femminile (dai 20 ai 64 anni) rimane tra i più bassi d'Europa: pur in risalita dopo gli anni della Grande Recessione, è arrivato nel 2017 solo al 52,5\%, con un divario con l'occupazione maschile tra i più alti, seppure - complice un maggiore tasso di disoccupazione maschile sceso al di sotto del $20 \%$. Nello stesso anno il tasso di occupazione femminile era in Spagna quasi del 60\% (con un divario di genere sotto $\mathrm{I}^{\prime} 11 \%$ ), in Francia quasi del $67 \%$ (con un divario sotto l' $8 \%$ ), in Svezia quasi dell' $80 \%$, con un divario di 4 punti" (p. 766). 
P01: [...] le madri sono più presenti, probabilmente perché non tutte lavorano, soprattutto le madri autoctone (insegnante di Educazione Artistica)

P02: Le mamme sono maggiormente presenti ai colloqui, alle riunioni e agli incontri vari perché hanno maggiore tempo a disposizione da trascorrere all'interno della scuola. (insegnante di Lettere, referente dell'area multiculturale)

Più in generale, I'aspetto degli impegni lavorativi che, stando allo scenario delineato dagli insegnanti, vedono maggiormente coinvolte le famiglie immigrate, rimanda a una riflessione più ampia. Innanzitutto, occorre partire dal ruolo degli immigrati nel mercato del lavoro italiano e alle differenze rispetto agli autoctoni. Si pensi, ad esempio, che il 31,3\% dei residenti extracomunitari, risulta impiegato nel settore dei servizi collettivi e alle persone (per lo più badanti, colf, babysitter e operatori domestici), mentre gli italiani impiegati nello stesso settore fanno registrare una percentuale pari al $5,2 \%{ }^{18}$. E, ancora, un migrante su 3 (il $36,5 \%$ del totale) accetta lavori umili, generalmente sottopagati, percentuale che tra gli italiani si riduce all' $8 \%{ }^{19}$. II tema delle diseguaglianze economiche, in sostanza, non solo nel nostro Paese continua ad essere rilevante, ma va ricordato anche che esso coinvolge in maniera rilevante proprio gli immigrati. Un'iniquità che può essere solo parzialmente corretta da politiche di redistribuzione e welfare (Saraceno e Saraceno, 2019). Su questo fronte, la scuola può incidere parzialmente, se non attraverso una funzione formativa che, ci si augura, possa implementare, seppur in maniera indiretta, le opportunità di mobilità sociale anche per gli studenti provenienti da famiglie immigrate. Allo stato attuale, però, è chiamata soprattutto ad adeguarsi, magari prevedendo servizi e agevolazioni che diano, in termini logistici, maggiori opportunità di partecipazione a tutti i genitori.

La presenza fisica, tuttavia, non è l'unica variabile su cui misurare il

18 Per un quadro complessivo, si consulti il VII Rapporto Annuale "Gli stranieri nel mercato del lavoro in Italia" (2017), da cui sono stati tratti i dati citati, disponibile on-line al sito:http://www.lavoro.gov.it/notizie/Pagine/Presentato-il-settimo-rapporto-annuale-Stranieri-in-mercato-lavoro-Italia.aspx.

19 II dato è tratto dal "XXVI Rapporto Immigrazione 2016" di Caritas e Migrantes, disponibile on-line al sito:

http://s2ew.caritasitaliana.it/materiali/Rapporto_immigrazione/2017/Sintesi_ RICM2016.pdf . 
Scuola e famiglie immigrate: Uno studio in una scuola del Sud Italia Marco Centorrino

coinvolgimento delle famiglie. Un'altra forma di condivisione - per gran parte dei docenti - è rappresentata dal supporto sociale e affettivo che questi nuclei manifestano nei riguardi dei propri figli. La strategia più ricorrente è l'iscrizione ad attività extrascolastiche, in modo che i ragazzi si relazionino con i loro compagni anche al di fuori della scuola.

P017: I ragazzi che ricevono supporto si notano subito, intanto perché vengono a scuola, da qui la motivazione propria all'apprendimento, all'integrazione sono stimolati a casa perché devono studiare, devono andare avanti. Allora tu vedi questi ragazzi molto attenti, molto timorosi se qualcosa non l'hanno svolta, partecipi, cercano di non sbagliare. I genitori, alcuni e non tutti, si mostrano interessati e cercano di fare in modo che $i$ loro figli frequentino attività extrascolastiche in modo da potersi relazionare con tutti gli altri compagni. (insegnante di Musica)

P011: [...] È importante motivare durante il percorso scolastico il processo di apprendimento degli alunni ed aiutarli nella misura in cui si può, incoraggiarli sempre più. Ciò vale soprattutto per quei ragazzi i cui genitori sono poco coinvolti nella vita dei propri figli. (insegnante di Scienze motorie)

Secondo diversi docenti la famiglia deve impegnarsi a sviluppare le relazioni sociali dei propri figli attraverso diverse iniziative, in particolare iscrivendoli nelle attività extrascolastiche che si organizzano a scuola (sportive, linguistiche-culturali, escursioni didattiche nelle quali partecipano insieme alle famiglie).

P027: [...]Molto spesso i genitori, coinvolti troppo nel loro lavoro, non pensano cosa sia giusto o non giusto per i propri figli, pertanto ritengo che le famiglie dovrebbero maggiormente sollecitare, incoraggiare i propri figli a dedicarsi ad attività extrascolastiche che sicuramente fanno bene da un punto di vista culturale, relazionale e, quindi, sociale. (insegnante di Religione)

P028: Ritengo che i genitori dovrebbero conoscere maggiormente i figli e per questo è importante il dialogo seguito da una partecipazione magari non assidua da parte della famiglia a scuola anche solo per conoscere i compagni del proprio figlio, I'andamento scolastico. Purtroppo ahimè alcuni ragazzi si sentono abbandonati, poco considerati dalla famiglia. (insegnante di Lettere) 
P030: A parer mio, l'aspetto integrativo è quello più importante ed è proprio esso che funge da collante e che si dovrebbe maggiormente sviluppare. Dico questo perché mi è capitato di notare insieme ad alcuni miei colleghi il fatto che diversi ragazzi stranieri tendono ad avere relazioni scolastiche ed extrascolastiche solo tra di loro ${ }^{20}$ e, d'altro canto, assumendo questo atteggiamento si allontanano dagli altri ragazzi autoctoni. Di conseguenza accade che in occasioni di feste di compleanno i ragazzi italiani non li invitano perché già percepiscono che per loro non sarebbe un piacere. Diciamo che in questo caso la famiglia dovrebbe intervenire, facendo capire a questi ragazzi stranieri che è importante relazionarsi con tutti senza differenze, ma spesso ciò non accade poiché anche le famiglie tendono a colloquiare maggiormente con quelle appartenenti alla loro nazionalità [...]. (insegnante di Religione)

Le osservazioni degli insegnanti tendono quindi a sottolineare la responsabilità per il da farsi (Jonas, 1979), cioè che obbliga ad azioni progettate per il benessere altrui, che la famiglia deve assumersi.

Negli ultimi anni scolastici, per consentire un maggiore coinvolgimento delle famiglie, si sono sviluppate una serie di iniziative inclusive in cui le famiglie stesse sono intervenute in maniera variegata. A questo proposito, riportiamo alcune delle affermazioni dei docenti intervistati:

P04: Tante famiglie, più locali che straniere, hanno partecipato alle attività, ai laboratori, al lavoro per progetti della scuola, grazie agli stimoli dettati dalla scuola. (insegnante d'Inglese)

P05: [...]Dai vari colloqui avuti so che diversi genitori immigrati hanno alcune idee molto creative, ma non prendono mai l'iniziativa per esporle per paura di sbagliare, di non sentirsi all'altezza o di essere poco adeguati al contesto. (insegnante di Matematica e Scienze)

P02: [...] Le attività promosse dalla scuola non sono tantissime, ma quelle realizzate cercano di fare in modo che le famiglie partecipino, collaborando tra di loro e apportando idee proprie e, ancor meglio, legate a tradizioni culturali differenti. Mi ricordo che proprio l'anno scorso la scuola ha deciso di promuovere una giornata dedicata al multiculturalismo etnico, in cui ogni fa-

20 In tal senso, appare utile ricordare come già Durkheim sottolineasse il ruolo svolto dalla scuola nella socializzazione dei ragazzi, poiché fa comprendere loro i valori comuni che trasformano una moltitudine di individui isolati in una collettività. 
Scuola e famiglie immigrate: Uno studio in una scuola del Sud Italia Marco Centorrino

miglia cucinava a casa un piatto tipico del proprio Paese e lo portava a scuola, ponendolo su un tavolo ben apparecchiato. Si potevano degustare tanti cibi diversi e ciò ha permesso a molte famiglie di conoscersi, di scambiarsi le proprie idee e tradizioni non solo gastronomiche. Ricordo in maniera nitida che quella giornata fu davvero apprezzata sia dai ragazzi sia dalle famiglie e si concluse con l'esibizione di ragazzi italiani che omaggiarono le famiglie immigrate con un canto in inglese. Viceversa, i ragazzi stranieri cantarono una canzone in lingua italiana. Da lì capii realmente che la scuola rappresenta un'istituzione importante, aggregante e che quindi funge da filo conduttore tra le varie culture, lingue, religioni e che le barriere non esistono, ma se le crea chi non ha mai vissuto realtà simili. (insegnante di Lettere, referente dell'area multiculturale)

P022: Dall'inizio dell'anno scorso abbiamo avuto il piacere di avviare il progetto intercultura denominato "L'arcobaleno di culture", rappresentato da una serie di laboratori diversi[immagine e teatro; scultura, disegno e lavori manuali; musica; lingua inglese; lingua italiana, alla riscoperta della poesia]. Tale progetto è principalmente rivolto agli alunni, ma è anche aperto alle famiglie e alla loro collaborazione. Infatti, devo dire che diverse famiglie, per organizzarsi e coordinarsi tra di loro, hanno creato un gruppo whatsapp. Si sono interessate al materiale da acquistare, si sono sempre messe in contatto con noi docenti per alcuni dubbi o anche riflessioni e consigli. Questi laboratori si svolgevano e si svolgono ogni 15 giorni, [...]. Tuttavia sia il giorno sia l'orario hanno sempre subito dei cambiamenti a seconda della disponibilità delle famiglie, affinché quelle che avevano maggiori difficoltà lavorative potessero parteciparvi. Le famiglie immigrate, però,erano e sono in forte minoranza [...]. (insegnante di Lettere)

Un altro ostacolo che impedisce il coinvolgimento delle famiglie immigrate, e non tanto di quelle autoctone, si riferisce alla presenza o meno di servizi pubblici e legati alle politiche di welfare e inclusione sociale. $\mathrm{Si}$ tratta di elementi che influiscono notevolmente nella relazione famiglia-scuola. Come si è già detto, uno dei fattori strutturali inteso come elemento di ostacolo riguarda l'assenza di una rete familiare allargata. Infatti, secondo qualche docente:

P09: [...] Le famiglie dei ragazzi stranieri non si presentano spesso ai colloqui non perché sono disinteressate all'istruzione del proprio figlio, ma 
perché non hanno il sostegno della rete familiare allargata: i nonni, gli zii, ecc.. Diversi genitori non partecipano spesso perché non sanno materialmente a chi affidare i ragazzi. Al contrario di ciò che avviene nelle famiglie autoctone ove il supporto della rete familiare è davvero ampio" (insegnante di Lettere)

P012: [...] Quasi sempre molte famiglie immigrate non sanno a chi affidare $i$ propri figli anche solo per andare a fare una visita medica e, quindi, sono costrette a portarli con sé. Altri, addirittura, li lasciano soli in casa seppur ancora minorenni." (insegnante di sostegno)

Alcuni docenti intervistati, poi, mettono in rilievo l'importanza di valorizzare le culture di origine dei genitori, come fattore che può facilitare la relazione e la collaborazione.

P031: [...] Alcune famiglie immigrate hanno voglia di raccontare la loro provenienza d'origine, gli studi compiuti, il conseguimento della loro laurea, la possibilità avuta nel poter vivere all'interno di una splendida casa accessoriata, non amano essere considerati solo per la situazione precaria attuale che vivono a Messina, ma per tutta la loro esperienza di vita, come un padre albanese che lavora a Villafranca, in provincia di Messina come muratore e nel suo paese era un docente. (insegnante di sostegno)

P032: In base alla mia esperienza scolastica ho da sempre notato che ci sono alcune famiglie come quelle albanesi e arabe che tengono tantissimo a far sapere che anche loro hanno studiato nei loro Paesi, ma che purtroppo l'istruzione personale non gli è bastata per poter andare avanti e, quindi, iniziano spesso durante le ore di ricevimento a raccontarmi il loro vissuto, ciò che avevano e che poi hanno perduto. Per questo nutrono alte aspettative nei riguardi del proprio figlio. (insegnante di Lettere)

P024: [...]ma sarebbe anche giusto valorizzare le culture di ogni famiglia, perché esse rappresentano un punto di forza. Infatti, la scuola non si permetterebbe mai di annullare la loro cultura e, quindi, la loro identità, anzi per la scuola la diversità culturale rappresenta un valore aggiunto, altrimenti non si potrebbe parlare della nostra scuola come scuola inclusiva sotto i diversi punti di vista. (insegnante di Matematica e Scienze) 
Pertanto si evince che i fattori culturali non costituiscono solo elementi di ostacolo, ma spesso fungono da risorsa nella relazione famiglia-scuola.

\section{Conclusioni}

Dalle interviste effettuate ci siamo resi conto di quanto sia importante e talvolta difficile riuscire ad instaurare una relazione reciproca che permetta un pieno coinvolgimento delle famiglie immigrate all'interno del contesto scolastico. Dietro agli sforzi messi in atto per favorire lo sviluppo di tale relazione, vi è l'obiettivo di far stare bene l'alunno, di favorire il suo sviluppo e fare in modo che possa integrarsi nella società nel migliore dei modi. Come si è potuto constatare, secondo i docenti, I'aspetto comunicativo fatto di ascolto e condivisione è importante per rendere efficace una relazione. Anche Garreta e Maciá (2017) affermano che la comunicazione deve essere bidirezionale per raggiungere una partecipazione efficace. Secondo Dubet (1997) è necessario stabilire un nuovo accordo tra famiglie e scuola per ricondurre a una situazione nella quale la scuola dovrebbe potenziare il coinvolgimento, i docenti mantenere il loro diritto ad esercitare liberamente e i genitori a difendere i loro interessi e quelli dei loro figli. Da quanto si evince dalla nostra indagine,un dato importante riguarda una partecipazione maggiore delle famiglie locali e minore di quelle immigrate. I fattori di ostacolo emersi dalle testimonianze dei docenti e che impediscono il coinvolgimento di queste famiglie all'interno del contesto scolastico possono essere riassunti così:

- La mancanza di un mediatore linguistico e culturale che faciliti l'integrazione e la partecipazione a partire dalla conoscenza, dalla comprensione e dall'uso corretto della lingua italiana;

- La mancanza di una formazione adeguata nelle lingue straniere tra un elevato numero di docenti;

- L'instabilità socioeconomica delle famiglie e, pertanto,la difficoltà a sostenere alcune spese;

- L'incompatibilità degli orari di lavoro con gli orari di apertura della scuola che non consentono alle famiglie immigrate di poter partecipare a riunioni, attività scolastiche e/o altri incontri;

- L'assenza di una rete familiare allargata nel territorio,che permetta alle famiglie di essere più presenti e partecipative a scuola. 
Sicuramente, questi fattori assumono un forte peso nel rendere più o meno efficace la relazione di partecipazione e pertanto di coinvolgimento con le famiglie immigrate.

Accanto a tutto ciò, vanno ovviamente tenute presenti alcune considerazioni legate agli studi sociologici di matrice funzionalista dedicati al concetto di istruzione, che possono costituire ulteriore elemento di ostacolo. Grazie ai sistemi educativi, infatti, gli individui acquisiscono le conoscenze e le competenze necessarie a vivere in società. Nei percorsi formativi della scuola si ritrovano, quindi, la morale e i valori sociali. Ciò con cui bisogna fare i conti, però, è il monolitismo dei valori sociali. Una società in cui si registrano resistenze ad ampliare la sfera dei valori fondamentali - basti pensare, in tal senso, ai tanti episodi di intolleranza a cui assistiamo quotidianamente - si riflette anche nell'istituzione scolastica e, appunto, sulla morale e sui valori sociali che essa propone. Sulla scia del pensiero conflittualista, va evidenziato che, nelle società contrassegnate da marcate disuguaglianze sociali, i sistemi educativi, in realtà, finiscono per rafforzarle e, in questo senso, I'istruzione scolastica fa gli interessi dei gruppi sociali dominanti.

\section{Bibliografia}

Abis, M. (2004). Background e motivi della ricerca: fra integrazione e integrazioni. Micro e Macro Marketing, 2, 421-427.

Baraldi, C. (2003). Comunicazione interculturale e diversità. Roma: Carocci.

Bennett, M. J. (a cura di) (2002). Principi di comunicazione interculturale, Milano: Franco Angeli.

Bochaca, J. G. (2008). Escuela, familia de origen inmigrante y participación. Revista de Educación, 345, 133-155.

Bolognesi, I. (2016). Coinvolgere i genitori nella scuola multiculturale. Un progetto di ricerca azione per la costruzione di una cultura della partecipazione. Rivista Italiana di Educazione Familiare, 2, 81-93.

Bonifazi, C. (a cura di) (2017). Migrazioni e integrazioni nell'Italia di oggi, Roma: Consiglio Nazionale delle Ricerche, Istituto di Ricerche sulla Popolazione e le Politiche Sociali.

Cartei, C. (2008). Una Scuola dell'infanzia accogliente, tra aspettative delle famiglie immigrate ed esperienze in atto. Rivista Italiana di Educazione Familiare, 2, 73-85.

Carzo, D. (2011). Discorsi e narrazioni sull'Altro: un'introduzione sul fenomeno immigratorio. In D.

Carzo (a cura di) Narrare l'Altro. Pratiche discorsive sull'immigrazione (pp. 11-18). Roma: 
Scuola e famiglie immigrate: Uno studio in una scuola del Sud Italia Marco Centorrino

Aracne.

Dandolo, F. (2018). Una rassegna sui temi dell'immigrazione in Italia.Rivista economica del Mezzogiorno, 1-2, 167-186.

De Santis, G. e Strozza, S. (a cura di) (2017). Rapporto sulla popolazione. Le molte facce della presenza straniera in Italia, Bologna: Il Mulino.

Di Bartolomeo, A., Bonifazi, C. e Strozza, S. (2017). Figli degli immigrati e riuscita scolastica. In C. Bonifazi (a cura di) Migrazioni e integrazioni nell'Italia di oggi (pp. 171-182), Roma: CNR-IRPPS e-Publishing.

Dubet, F. (1997). École, familles: le malentendu. Paris: Les Editions Textuel.

Favaro, G. (2010). Una lingua "seconda e adottiva". L'italiano delle seconde generazioni. Italiano Lingua Due, 1, 1-14.

Garreta, J. y Maciá, M. (2017). La comunicación familia-escuela. In J. Garreta (coord.) Familias y escuelas: discursos y prácticas sobre la participación en la escuela (pp. 7198). Madrid: Ediciones Pirámide.

Granata, A., Mejri, O. e Rizzi, F. (2015). Non è solo questione di cultura. Fattori di ostacolo e risorse nella relazione famiglia-scuola dell'infanzia. Rivista Italiana di Educazione Familiare, 10, 77-91.

Grasso, M. (2015). Il successo scolastico dei giovani figli dell'immigrazione: il ruolo della famiglia come capitale sociale. Rivista italiana di educazione familiare, 1, 201-216.

Gudykunst, W.B. e Mody, B. (eds) (2002). Handbook of International and Intercultural Communication, Thousand Oaks: Sage.

Jonas, H. (1979). Das Prinzip Verantwortung. Francoforte: Verlag (trad. it. II principio di reponsabilità. Un'etica per la civiltà tecnologica. Torino: Einaudi, 1990).

Mencarini, L. (2018). Padri e madri, lavoro e famiglia: un Paese non al passo coi tempi. il Mulino, 5, 766-773.

Molinari, L. e Bertocchi, S. (2007). La responsabilità a scuola e in famiglia. Una ricerca su insegnanti e genitori. Giornale italiano di psicologia, 4, 901-920.

Mucchi Faina, A. (2006). Comunicazione interculturale. Il punto di vista psicologico-sociale. Roma-Bari: Laterza.

Rogoff, B. (2003). The Cultural Nature of Human Development. Oxford: Oxford University Press (trad. it. La natura culturale dello sviluppo. Milano: Raffaello Cortina, 2004).

Saraceno, P. e Saraceno, C. (2019). Contro le disuguaglianze insostenibili: non solo Welfare.il Mulino, 2, 254-261.

Silva, C. (2009). La relazione tra genitori immigrati e insegnanti nella scuola dell'infanzia. Rivista Italiana di educazione familiare, 3, 23-36.

Zoletto, D. (2007). Straniero in classe. Una pedagogia dell'ospitalità. Milano: Raffaello Cortina. 


\section{Appendice}

\section{Tabella 1}

Distribuzione degli alunni stranieri e autoctoni nelle classi della Scuola Secondaria di I grado dalla prima alla terza dell'istituto "Manzoni-Dina e Clarenza" di Messina.

\begin{tabular}{ccccccc}
\hline & \multicolumn{2}{c}{ Prime Classi } & \multicolumn{2}{c}{ Seconde Classi } & \multicolumn{2}{c}{ Terze Classi } \\
& $2018-2019$ & $2018-2019$ & $2018-2019$ \\
\hline Nazionalità & $N$ & $\%$ & $N$ & $\%$ & $N$ & $\%$ \\
Rumena & 3 & 2,08 & 1 & 0,80 & 0 & 0,00 \\
Filippina & 4 & 2,78 & 2 & 1,60 & 2 & 1,94 \\
Srilankese & 2 & 1,39 & 3 & 2,40 & 1 & 0,97 \\
Cinese & 1 & 0,69 & 0 & 0,00 & 2 & 1,94 \\
Senegalese & 0 & 0,00 & 0 & 0,00 & 0 & 0,00 \\
Bangladese & 0 & 0,00 & 1 & 0,80 & 0 & 0,00 \\
Italiana & 129 & 89,58 & 111 & 88,80 & 96 & 93,20 \\
Marocchina & 3 & 2,08 & 3 & 2,40 & 1 & 0,97 \\
Gambiana & 0 & 0,00 & 1 & 0,80 & 0 & 0,00 \\
Ivoriana & 0 & 0,00 & 1 & 0,80 & 0 & 0,00 \\
Sierraleonese & 0 & 0,00 & 1 & 0,80 & 0 & 0,00 \\
Ucraina & 0 & 0,00 & 0 & 0,00 & 0 & 0,00 \\
Statunitense & 1 & 0,69 & 1 & 0,80 & 1 & 0,97 \\
Brasiliana & 1 & 0,69 & 0 & 0,00 & 0 & 0,00 \\
\hline Total & 144 & 100,00 & 125 & 100,00 & 103 & 100,00 \\
\hline
\end{tabular}

Fonte: Elaborazione propria

Legenda: $\mathrm{N}=$ numero alunni; $\%=$ percentuale sul totale 
